\title{
Making molecules vibrate: Interactive web environment for the teaching of infrared
}

\section{spectroscopy}

\author{
Kevin Maik Jablonka, ${ }^{+, \mathbb{I}}$ Luc Patiny, ${ }^{\ddagger,}$ II and Berend Smit*,+ \\ †Laboratory of Molecular Simulation (LSMO), Institut des Sciences et Ingénierie Chimiques, École \\ Polytechnique Fédérale de Lausanne (EPFL), Rue de l'Industrie 17, CH-1951 Sion, Valais, \\ Switzerland \\ $\ddagger$ Institut des Sciences et Ingénierie Chimiques, École Polytechnique Fédérale de Lausanne (EPFL), \\ Lausanne, Switzerland \\ IThese authors contributed equally. \\ E-mail: berend.smit@epfl.ch
}

\begin{abstract}
Infrared spectroscopy (IR) is a staple structural elucidation and characterization technique due to its ability to identify functional groups and its ease of use. Interestingly, it allows capturing electronic effects via their influence on the bond strength of "probes" such as the carbonyl group and also offers a wealth of examples for discussion on the theory of electronic transitions. For this reason, IR spectroscopy is typically taught both in theoretical classes and in applied structural analysis courses. In practice, there is rarely a link between those courses, and both suffer from the lack of exploratory learning, i.e., tools with which students can explore the interplay between symmetry and selection rules, as well as electronic effects and vibrational frequencies - with almost immediate feedback. In practice, this might lead to students that are well skilled
\end{abstract}


in looking up vibrational frequencies in lookup tables but do not understand the links to electronic effects and reactivity. Here, we introduce a web app that leverages semiempirical quantum mechanical (or force-field based) calculations, performed on a web service, in an interactive interface to provide an environment in which students can explore how slight changes to the structure manifest in changes of the spectrum. This approach avoids the time-consuming handling of potentially hazardous materials that might not be readily available and invites students to play with spectroscopy — to "see" and "test" electronic effects that are so commonplace in organic chemistry education. As a "side effect" our web app also provides a powerful aid for research scientists to investigate how different structural modifications, such as substitution, isomerism, or steric strain, would manifest in the infrared spectrum.

\section{Introduction}

Infrared (IR) spectroscopy is a cornerstone technique for structure elucidation. For decades, organic chemists based much of their characterization efforts on it. For instance, Woodward wrote that "infrared spectra have now become an asset of almost incomparable value to all chemists interested in structural problems in organic chemistry". 11 But also in inorganic chemistry, IR spectroscopy is an indispensable tool, where, for example, the carbonyl stretching frequencies are often used to probe the structure and electronic configuration of metal centers. ${ }^{[2}$ Due to its importance in analytical chemistry, IR spectroscopy is an essential component of the chemistry curriculum and is often thought in structural analysis — where the focus is typically to learn the mapping from tabulated group frequencies to structures. However, it is also an essential component of theoretical spectroscopy classes - where the focus is often on the derivation of selection rules. Such a separation of IR spectroscopy into a very practical and theoretical part has the risk that students have difficulties integrating the two aspects. Students that can match spectral signatures to functional groups (using correlation tables) or are able to evaluate the transition dipole moment integral often fail to 
provide basic physical reasoning for why for their particular compound a certain vibration might be forbidden or shifted by substitution, or how vibrational frequencies are related to reactivity and electronic effects. This lack of deep, intuitive, understanding will manifest itself in students not being able to solve real-live research questions. ${ }^{3}$

These problems have motivated educators to better integrate IR spectroscopy in the curriculum, $\frac{455}{45}$ for example via example spectra,,$\frac{13}{3}$ by using computational techniques for the specific case of alkynes, ${ }^{677}$ via machine learning exercises, ${ }^{8}$ or virtual reality. ${ }^{9}$ However, there is still a lack of an integrated, customizable, and interactive, combination of theoretical, computational, and experimental aspects that is not "hard-coded" for a specific case study (and hence could also be used in a research environment) and provides immediate feedback on hypotheses formulated by a student.

Here, we report an interactive web tool that allows students to simulate and analyze IR spectra for arbitrary (organic) molecules. The vibrational modes are animated and interactively coupled to the spectrum and structural drawing. Students can hover over bands in the spectrum and discover the relevant modes, bonds, and atoms. Via the introduction of small substitutions, students can formulate and test hypotheses, for example on the gross selection rules, and investigate how the electronic structure, i.e. changes in the electron density distribution caused by functional groups, influences the vibrational frequency. In quite a similar way, they can also use the tool in synthetic laboratory courses - or actual research — to aid the interpretation of their IR spectra. To guide the students' exploration our web app also provides to option to create curated collections of molecules that, for example, could be used to showcase electronic effects such as the mesomeric and inductive effect. The tool can be used via our public website (https://go.epfl.ch/learnir) or hosted on a local machine. 


\section{Making molecules vibrate}

A detailed description of the technical aspect of the framework of our web-based tool is provided in the Method section. Here, we provide a basic description of the process.

Our framework is built from two main components. First, a Python-based web service performs the conformer search, and geometry optimization, and then simulates the IR spectrum using different levels of theory (force fields or semi-empirical quantum chemistry). This web service, which we deploy publicly on one our servers (https://ir.cheminfo.org/api/v1/docs), is not directly queried by the students but can be used via a web interface we developed, in which interactive visualizations invite to explore the relationship between structures and spectra.

In an editor pane, students can draw any molecule of their choice and select the level of theory (in the public deployment we impose a limit on the number of atoms due to finite computational resources). A click on the calculation button launches the webserver and the computed spectrum will appear in another pane together with a table filled with all modes. The modes can be selected via clicking on a band in the spectrum or a row in the table and will then be animated. Additionally, also clicks on a bond will be interactively linked to the animation of the most relevant mode. An important aspect of this tool is that the student can make changes in the structure and the feedback in form of a new simulated spectrum is only in the order of seconds. This instantaneous feedback has been lacking in the currently available tools, and as we will show in the case studies, this invites students to interactively explore hypotheses and improve their intuition and understanding.

As a particular feature for teaching, the interface also gives the option to superimpose spectra - for example to investigate how the frequency of certain vibrations is related to the structure. To structure the exploration of such relationships, the app also gives the possibility to load customizable libraries of predefined compounds which, for example, could be curated by the teacher as a starting point for the exploration of certain concepts. 


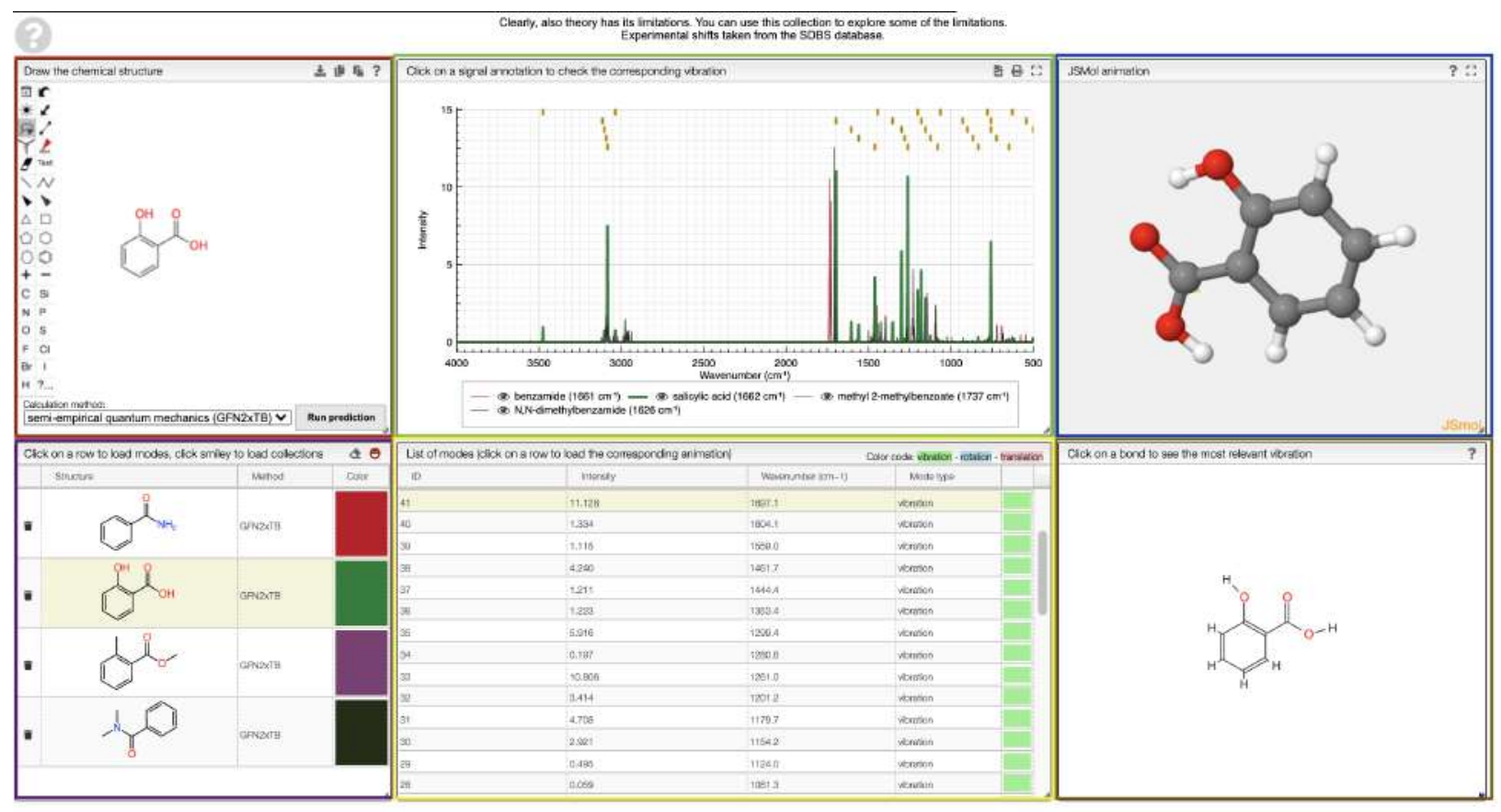

Figure 1: The main web interface. All components are interactive. Upon the first visit, a short tour guides the user through the main interfaces and options, which are additionally documented in the "Help menu" (question mark icon). A structural editor (red highlight) allows to modify a pre-loaded structure or to draw a completely new structure. The normal modes are tabulated (yellow highlight) and linked to a graph of the IR spectrum (green highlight) and interactive visualization of the vibrational mode (blue highlight). An interactive 2D drawing (brown highlight) allows discovering which mode is most relevant for a given bond (and the mode will be highlighted in the table and animated upon click). The spectrum can be simulated for an arbitrary number of molecules, which can then be superimposed and appear in distinct colors in the plot and an interactive table (violet highlight) allows to toggle between molecules. 


\section{Some example case studies}

In the following case studies, we illustrate a few such potential exercises which are also part of the default structure libraries provided in the app.

\section{Exploring the gross selection rule}

The concept: A key result in courses on theoretical spectroscopy is that a vibrational mode can only be observed if it is connected to a change in dipole moment. This result is deeply connected to molecular symmetry and the IR active modes can be derived using group theoretic considerations. $10-12$

Didactic challenges: From a didactic point of view, the issue here is that students have to apply multiple (new) abstract concepts at the same time, which for many students can lead to a cognitive overload. ${ }^{13}$ Students not only need to translate a chemical formula into a three-dimensional structure, but also visualize the vibrations and analyze if there would be a change in dipole moment - while keeping the selection rule in mind. Our tool aims to reduce this cognitive load by enumerating and animating the vibrations such that students can focus on the application of the selection rule.

Interactive examples: We developed some case studies to explore this concept. For example, the instructor can ask the students to think about the vibrational modes of $\mathrm{O}=\mathrm{C}=\mathrm{O}$ and $\mathrm{O}=\mathrm{C}=\mathrm{S}$, as well as of $\mathrm{CH}_{4}$ and $\mathrm{CBrClFI}$ and then compare with the simulations, particularly analyzing the predicted vibrational intensities.

In the frequently discussed example of the $\mathrm{O}=\mathrm{C}=\mathrm{O}$ vs. $\mathrm{O}=\mathrm{C}=\mathrm{S}$ stretching frequency, for example, students will find no intensity - i.e., IR activity - for the symmetrical $\mathrm{CO}_{2}$ stretching frequency whereas they will find that the same mode for COS is IR active. These observations can then be rationalized by the insight that there must be a change in the dipole moment for a vibrational mode to be IR active. 
Follow-up exercises: Using the interactive tool, students are not limited to simple linear or tetrahedral test cases but can test their understanding by designing complex pairs of molecules for which some modes are IR inactive, and directly get feedback on their ideas. At a more advanced level, this exercise can also be nicely coupled with an analysis of the irreducible representation of the point group, which, for example, for the $\mathcal{T}_{d}$ case would show that only the modes of ${ }^{2} T_{2}$ have the right symmetry to be IR active - and students could use the web app to confirm their group-theoretic analysis.

\section{Using carbonyls as a probe for electronic effects}
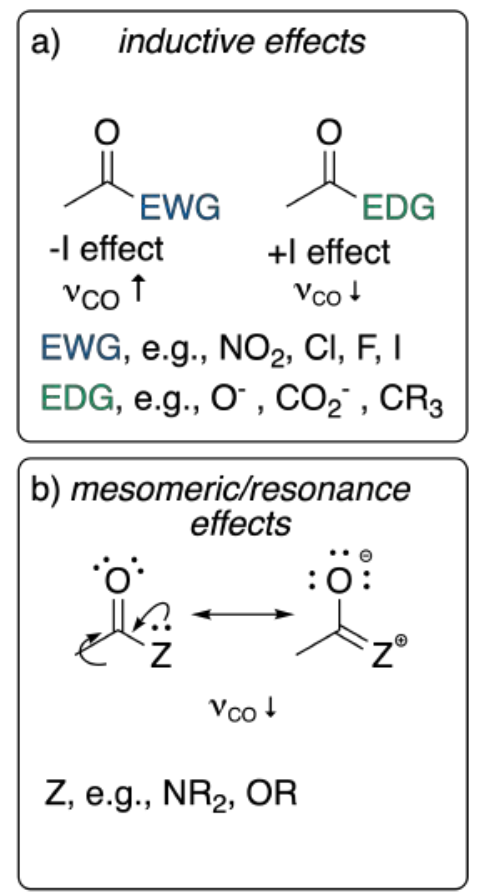

Figure 2: Inductive vs. mesomeric effects. The same group might show both behaviors (in opposite directions in terms of vibrational frequency). EWG, electron-withdrawing group; EDG, electro-donating group.

The concept: Electronic effects such as the mesomeric or inductive effect (Figure 2) are often used to rationalize regioselectivity — for example, the preference for certain 
sites for aromatic substitutions in the presence of "directing groups" - or reactivity w.r.t. nucleophilic substitution.

The didactic challenge: These inductive effects, however, can often be rather abstract, and in some cases, it is difficult to disentangle the relative importance of competing effects. Moreover, there is a strong interplay with other effects such as steric crowding or ring strain. Additionally, it is also relatively hard for students to actively do and explore something that gives direct feedback on these electronic effects - that is, there is a lack of inquiry-based approaches for teaching these concepts. Our case study was inspired by the work of McEvoy, 14 spectrum of different aromatic compounds containing a carbonyl group. Our tool can, of course, not replace a lab exercise, in which the students learn to handle the different substances and learn the effort and time it takes to get an experimental result. However, such an experimental environment is not always accessible to all students. In our web app, there is of course an additional advantage of the almost immediate feedback, and the app is allowing students to "play" with the chemistry - even for compounds that are unlikely to be available in any chemical catalog. The learning objectives are to (i) develop an intuitive understanding of the relation between structure and IR spectrum, (ii) develop a conceptual understanding of the relationships between vibrational frequencies, electronic effects, and reactivity.

\section{Inductive effect}

The inductive effect goes back to pioneering work from Hammett in which he shows that the substituent on a benzene ring can drastically influence the rate of the reaction. ${ }^{15}$ This concept is now widely used to explain reactivities in terms of the electron-donating or electron-withdrawing character of the substituents. In the case of the carbonyl group, for example, electron-withdrawing groups (EWG) "withdraw" charge from the carbonyl 
carbon, making it more positive, and hence susceptible for a nucleophilic attack. The important insights students can gain from our tool is that the change in the partial charge of the carbon atom is reflected in the bond strength (which can be thought of as changing the force constant) and hence manifests itself in changes in the carbonyl stretching frequency. The carbonyl group can hence be used to probe the strength of the inductive effect of different groups (also in complicated substitution patterns) in an interactive fashion. Figure 3 illustrates this at the example of benzaldehyde derivates where one can see that an increase in electronegativity increases the carbonyl stretching frequency.

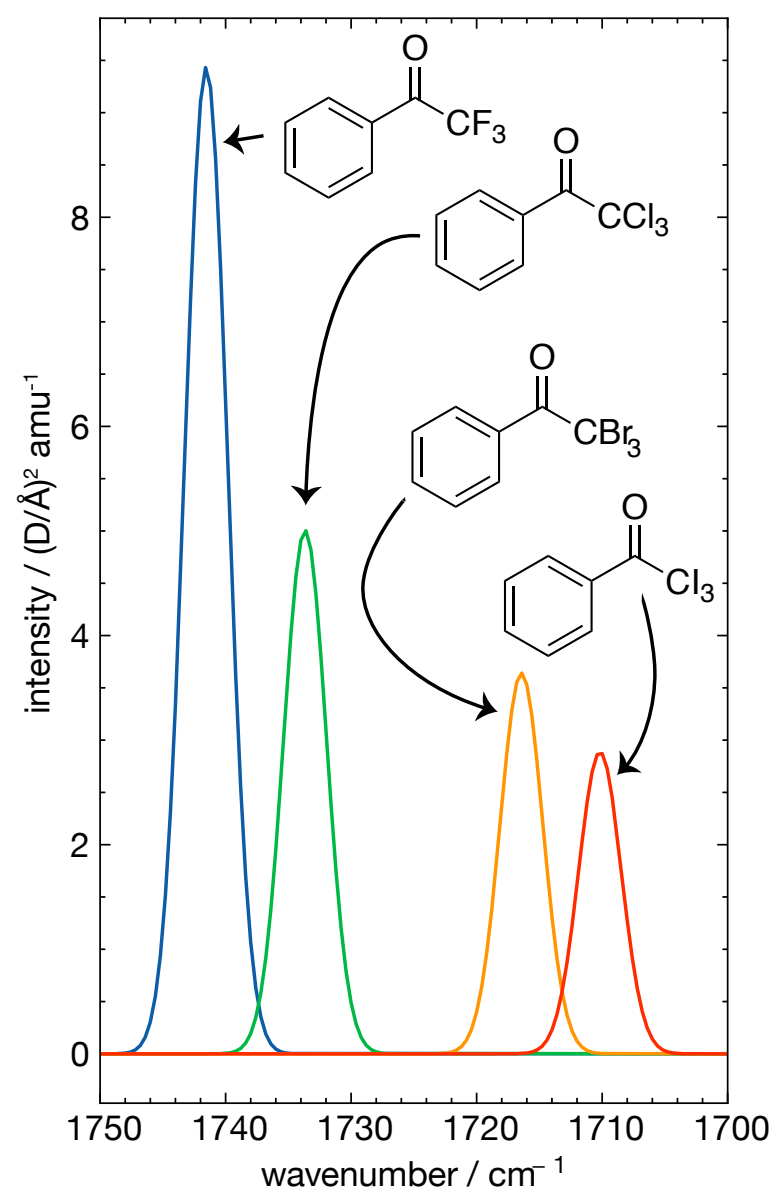

Figure 3: Carbonyl stretching frequency as a probe for inductive electronic effects. Changing the electronegativity of the substituent changes, via the inductive effect, the strength of the carbonyl bond, which is reflected in a change of the stretching frequency. 


\section{Mesomeric effect}

An effect that is sometimes competing with the inductive effect is the mesomeric effect. Similar to the inductive effect, it can be thought of as changing the electron density and hence influencing the reactivity. In the case of the mesomeric effect, the interaction of $\pi$ electron systems leads to the delocalization of electron density. As can be seen in Figure 2 b) the carbonyl bond is weakened - which can also be visualized via the partial charge not being on the carbonyl carbon but rather on the $\mathrm{Z}$ substituent. Also this change is reflected in the IR spectrum. However, we would expect that the trend is opposite to the one for the inductive effect, lowering the vibrational frequency due to a decrease in the bond strength. As shown in Figure 4 , the shifts simulated with our app support this hypothesis.

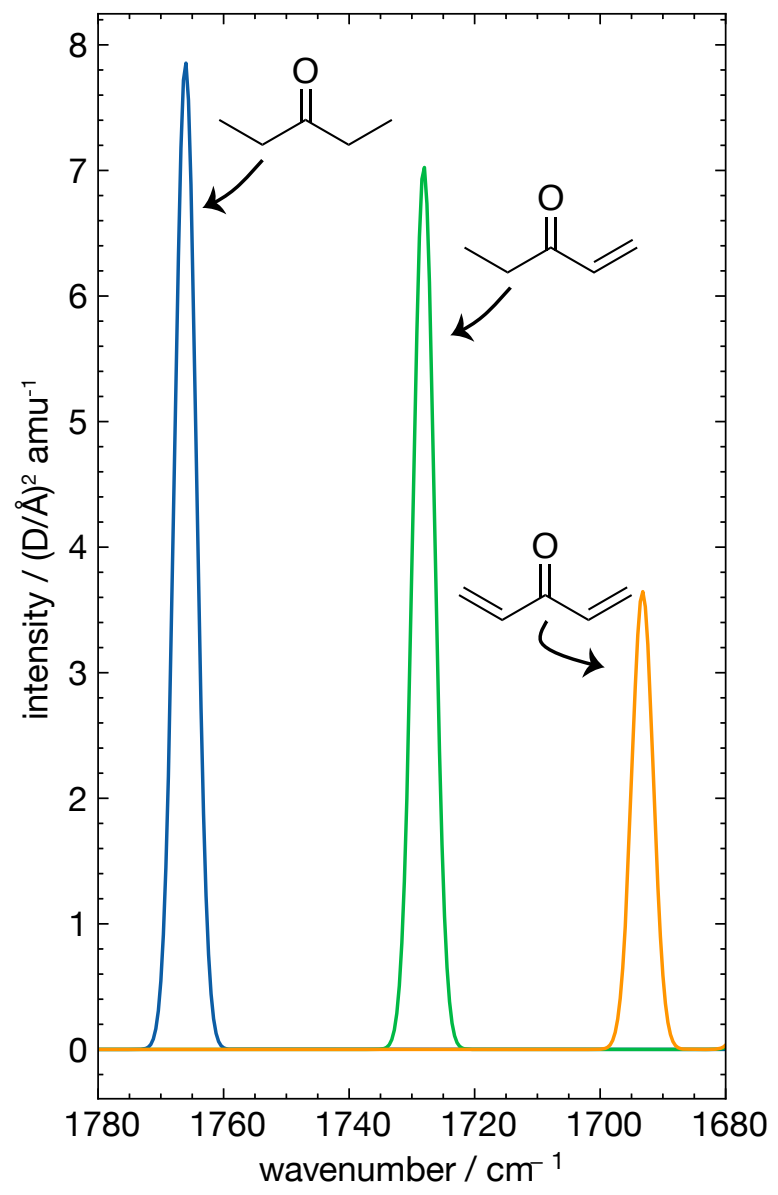

Figure 4: Carbonyl stretching frequency as a probe for the mesomeric effect. Delocalization weakens the $\mathrm{C}=\mathrm{O}$ bond and hence lowers the vibrational frequency. 


\section{Competing electronic effects}

The ester functional group is a prime example where one can enumerate competing ( $\sigma$, $\pi$ ) electronic effects on the strength - and hence vibrational frequency - of the carbonyl bond. ${ }^{16}$ In particular, one can imagine (i) a $\pi$ donation into the $\mathrm{C}=\mathrm{O} \pi^{*}$ acceptor, (ii) a $\sigma$ donation effect from the lone pair into the $\mathrm{C}=\mathrm{O} \sigma^{*}$ acceptor, (iii) a donation from the carbonyl lone pair into the $\mathrm{C}-\mathrm{O} \sigma^{*}$ acceptor (Figure $5 \mathrm{a}$ ). The occupation of antibonding orbitals $\left(\pi^{*}, \sigma^{*}\right)$ weakens, as the name suggests, bonds. Hence, for the first two effects, one would expect that they yield a lower wavenumber, whereas the latter one should increase the wavenumber (which is proportional to the ratio of the force constant, $k$, and the reduced mass $\mu: \tilde{v} \propto \sqrt{k / \mu}$ ). From the simulation of the IR spectrum, we get a direct indication that the last effect wins over the first two (see Figure 5). However, the beauty of our web app is that students do not need to stop there. Students can tune the ratio of the different effects by performing small changes to the structure, e.g., replacing oxygen with the less electronegative and more diffuse sulfur and selenium (Figure 5 b). From the simulated vibrational frequencies (Figure $5 \mathrm{~b}$ ) students can then see that the biggest increase in force constant comes from the acetyl fluoride, whereas the biggest weakening due to resonance effects - comes from the amide.

\section{Relative position of substituents}

One of the main learning objectives of lectures on organic synthesis is a deep understanding of how so-called directing groups can influence the outcome of syntheses. For example, comparing the ortho, meta, and para forms of nitro-benzaldehyde, the reactivity of the aldehyde group varies with the relative position with respect to the aldehyde (Figure 6). In a classroom setting, students can be asked to rationalize this observation using resonance structures and compare it with the one for a system with a donating group such as methoxy (Figure 7). Similarly, students can easily compare the directing strength of 
a)

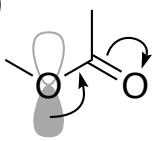

(i) $\pi$ donation

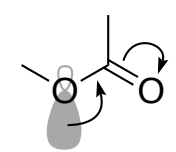

(ii) $\sigma$ donation c) $\begin{aligned} & 16 \\ & 14\end{aligned}$

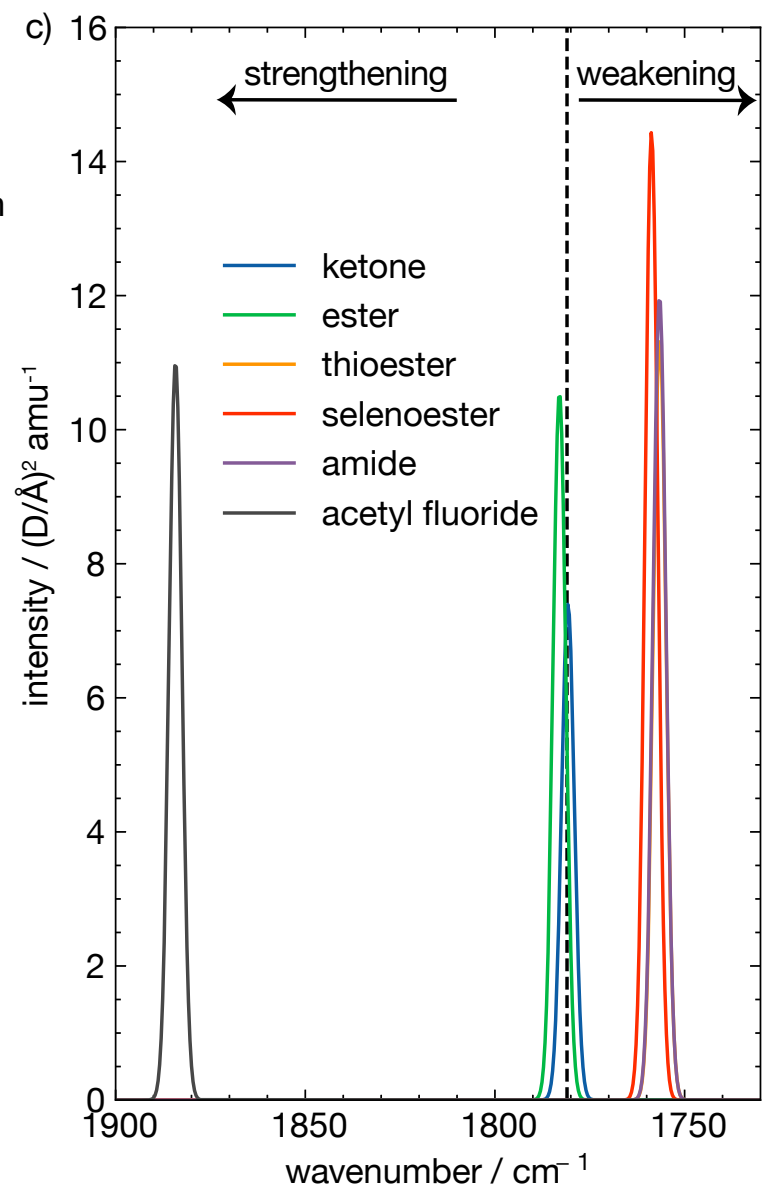

b)

(iii) $\sigma$ acceptance<smiles>COC(C)=O</smiles><smiles>CSC(C)=O</smiles>

ester thioester<smiles>C[Se]C(C)=O</smiles><smiles>CCC(C)=O</smiles>

selenoester

ketone<smiles>CNC(C)=O</smiles>

amide acetyl fluoride<smiles>CC(=O)F</smiles>

Figure 5: Using the carbonyl stretching frequency as a probe for electronic effects. a) Illustration of the electronic effects one can imagine to be relevant for an amide. b) Structures for which we computed the IR spectrum. c) The dashed line indicates the ketone $\mathrm{C}=\mathrm{O}$ stretching frequency as reference. Some substitutions stiffen the $\mathrm{C}=\mathrm{O}$ bond and hence cause a shift towards a larger wavenumber. Others soften the $\mathrm{C}=\mathrm{O}$ bonds, leading to a lower wavenumber. 
different substituents by simply replacing them and also analyze the behavior of more complicated substitution patterns with ease.

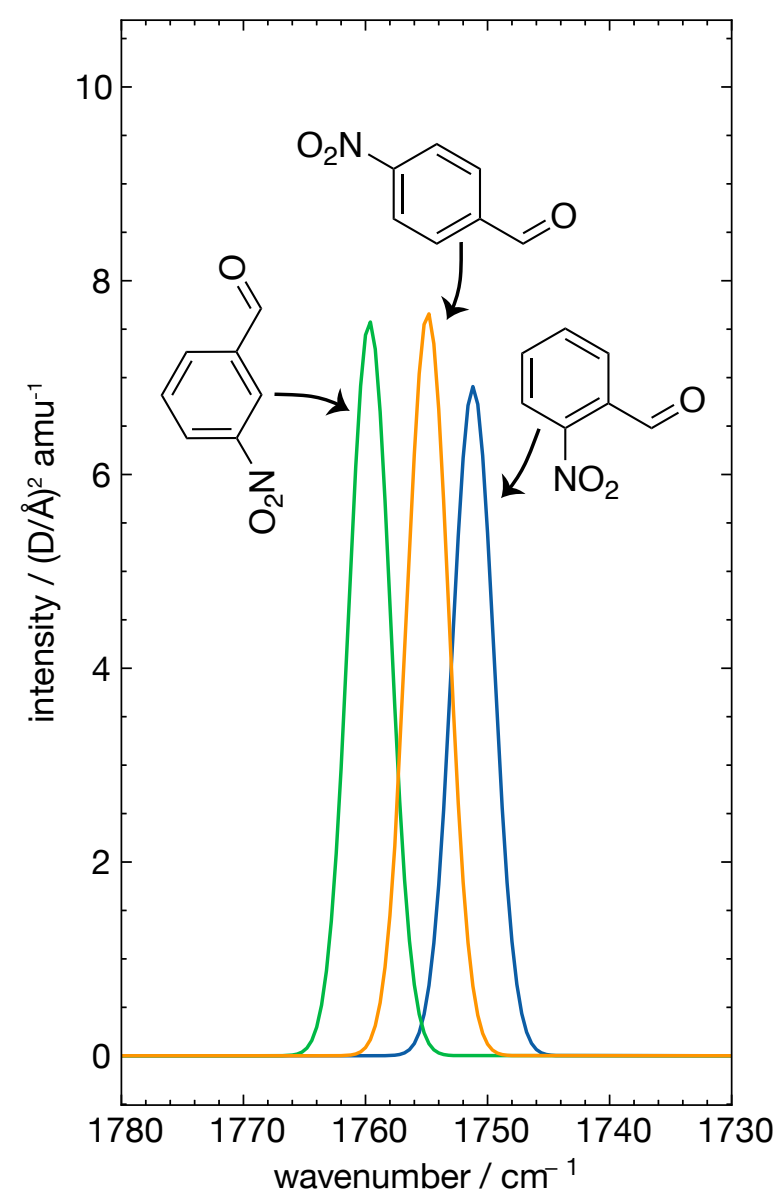

Figure 6: Using carbonyl as a probe for the meta-directing effect of electron-withdrawing substituents.

\section{Using the carbonyl stretching frequency as a probe for steric and strain effects}

The concept: The strength of electronic effects depends on how readily energetically similar orbitals can interact. This is clearly related to the 3D geometry of structures. Steric crowding can, for example, lead to a geometry in which the $\pi$ orbitals no longer properly align — which will lead to a less pronounced mesomeric effect. Similarly, ring strain 


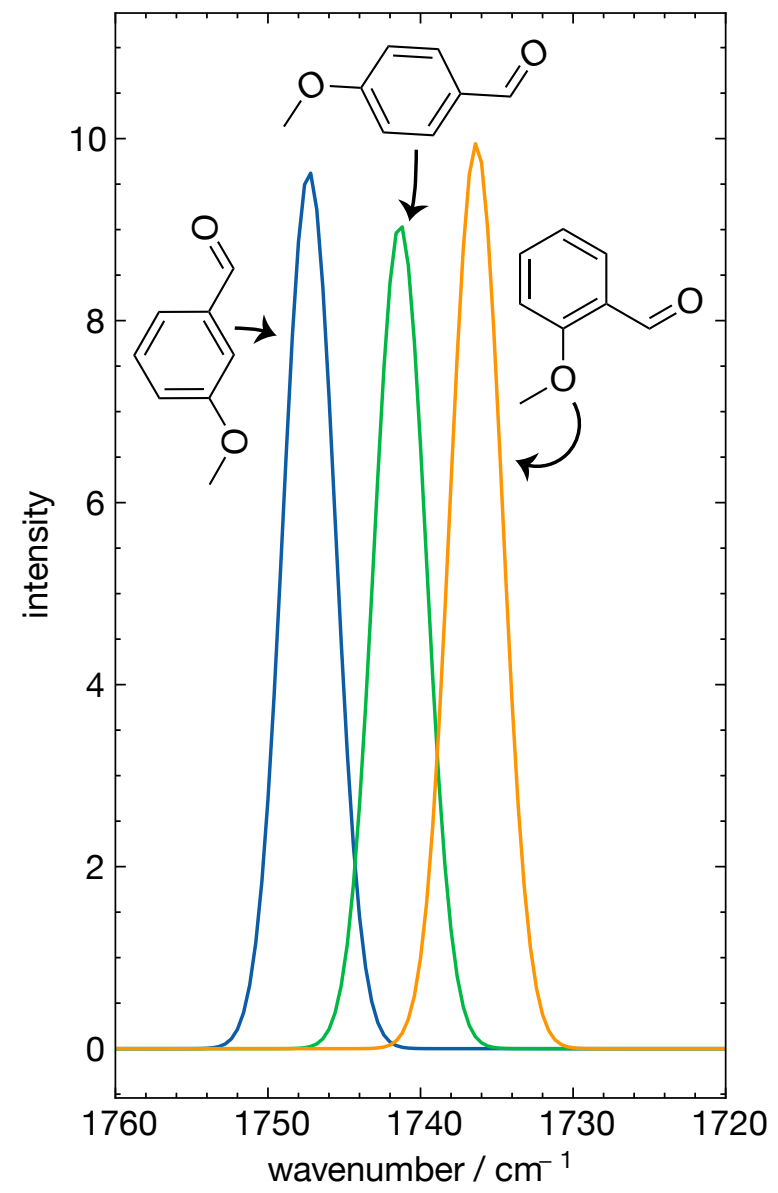

Figure 7: Using the carbonyl stretching frequency as a probe for the ortho/para-directing effect of electron-donating substituents. 
imposes a change in the $\mathrm{R}_{1}-(\mathrm{C}=\mathrm{O})-\mathrm{R}_{2}$ angle which also manifests itself in changes in the electron density of the carbonyl bond.

The didactic challenge: Also these phenomena can lead to a too high cognitive load for students. Students do not only need to visualize how the three-dimensional structure is changed by substituents but also connect this to changes in the electronic structure and then correlate this to changes in the IR frequency (or reactivity). Our web app lowers the cognitive load by providing visualizations of the equilibrium geometry and, additionally, invites the students to use an inquiry-based approach and test the effect of new, hypothetical, substitutions.

\section{Steric effects}

In Figure 8 we give an example of how the varying steric demand of functionalizations changes the carbonyl stretching frequency. In the figure, we show, in addition to the two-dimensional structural drawing, the three-dimensional relaxed structure. From the three-dimensional structures, it is clear that while the $\pi$ systems of the $\mathrm{C}=\mathrm{C}$ and $\mathrm{C}=\mathrm{O}$ bond are in the same plane for the (Z)-2,2-dimethylhex-4-en-3-one the sterical demands of the $\left(\mathrm{CH}_{3}\right)_{3}$ and $\mathrm{Ph}_{3}$ systems introduce a non-zero dihedral angle between the $\pi$ systems, which leads to a reduction of the strength of the mesomeric effect - and hence carbonyl stretches at lower wavenumbers.

\section{Ring strain}

One of the most important insights of chemistry in the 20th century was Baeyer's strain theory that explained the relative stabilities of cyclic molecules with different ring sizes (Nobel prize 1905). ${ }^{17}$ The core idea here is that a deviation from the "optimal" bonding environment causes a "strain" in the structure - which can be used to drive reactions such as ring-opening polymerizations. To understand the effects shown in Figure 9 


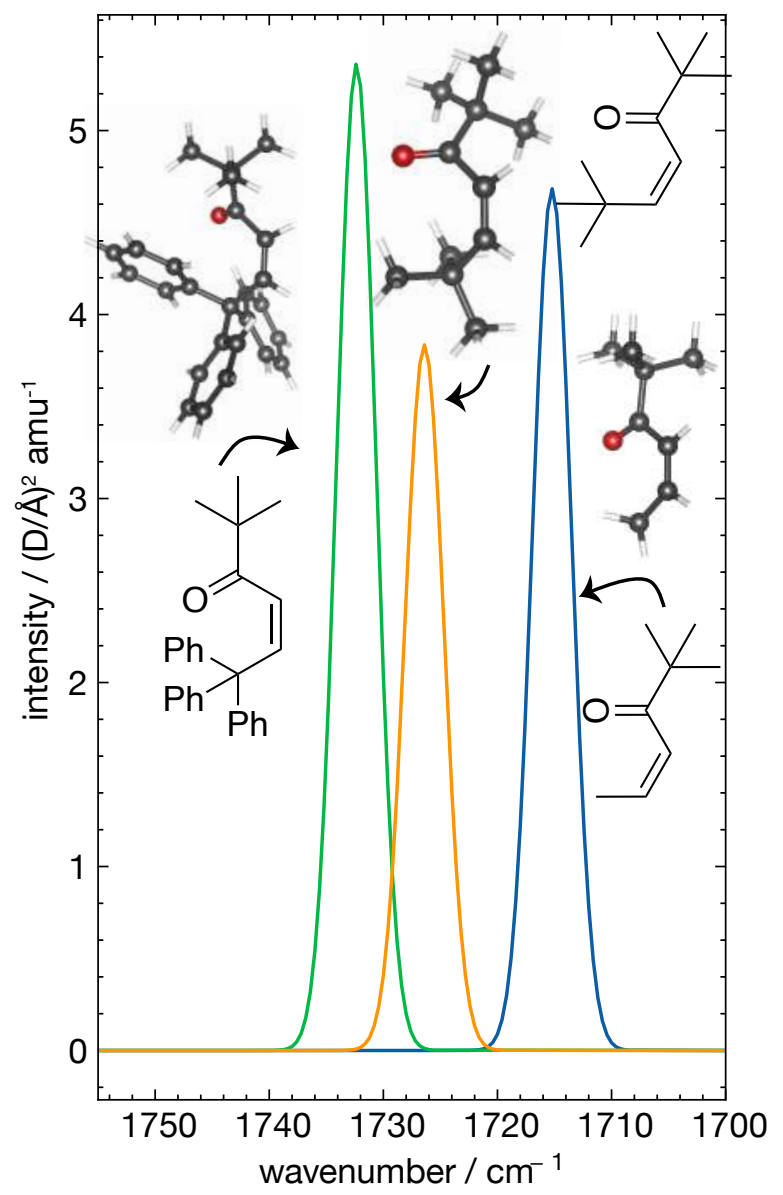

Figure 8: Using the carbonyl stretching frequency as a probe for steric effects. Here, sterically demanding substituents prevent the interaction of the $\mathrm{C}=\mathrm{O}$ and $\mathrm{C}=\mathrm{C} \pi$ systems. This leads to a reduction of the strength of the mesomeric effect, which is reflected in the stretching frequency of the carbonyl group. 


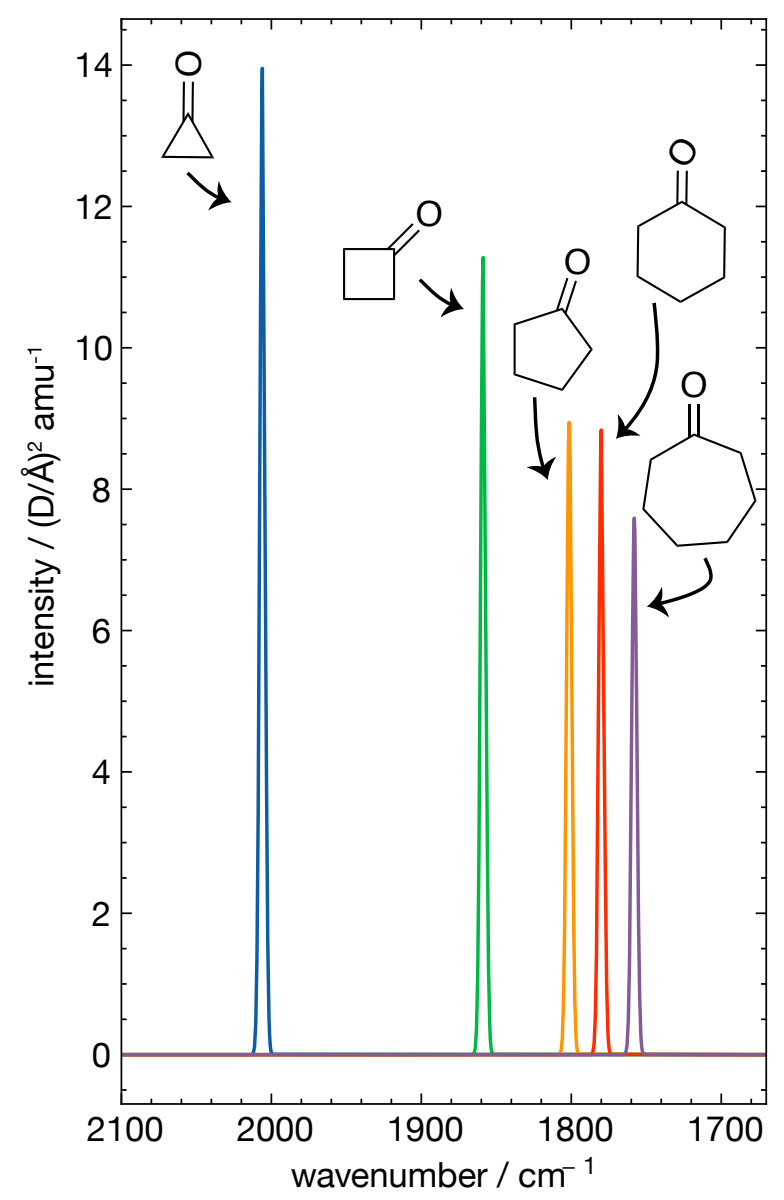

Figure 9: Using the carbonyl stretching frequency as a probe for ring strain. Smaller removing $\mathrm{CH}_{2}$ groups increases the $\mathrm{C}=\mathrm{O}$ stretching frequency. 
it is instructive to consult the animations (see snapshots in Figure 10). For the small cyclopropane ring students will see that the $\mathrm{C}=\mathrm{O}$ stretch requires are relatively large change in the length of the neighboring $\mathrm{C}-\mathrm{C}$ bond. This strong compression manifests itself in a large force constant — and hence absorption at higher wavenumbers. $\frac{18}{18}$

Interestingly, this exercise also reflects another important concept, namely that not only a single pair of atoms is involved in a normal mode but rather all atoms. This is, for example, clear if one analyzes the compression of the $\mathrm{C}-\mathrm{C}$ bond opposite to the carbonyl group in the cyclopropanone.

\section{Training inquiry skills: Limitations of theory}

The examples we have shown so far highlight the successes of the theory, even to the risk that students may get a too optimistic view of what modern theory can do. To stimulate the students' inquiry skills and their critical thinking skills, it is important to identify and test possible problems, e.g., limitations of a theory. In the case of our web app, an important limitation is the inability of the force-field or semi-empirical method to describe hydrogen-bond effects. Students can test this by comparing experimental data with computational results, where they will observe larger deviations for functional groups that can be involved in hydrogen bonding (see Figure 11), which then can lead to the hypothesis that the model cannot describe this effect (given that we simulate an isolated molecule in vacuum and not the molecular packing).

\section{Future work}

We are currently investigating the option to compute the IR spectrum as Boltzmann average of an ensemble of conformers. While this option is not relevant for the default teaching case, it can yield insight into one contribution to spectral broadening. Additionally, we are investigating the extension of our infrastructure to periodic systems. However, the larger 

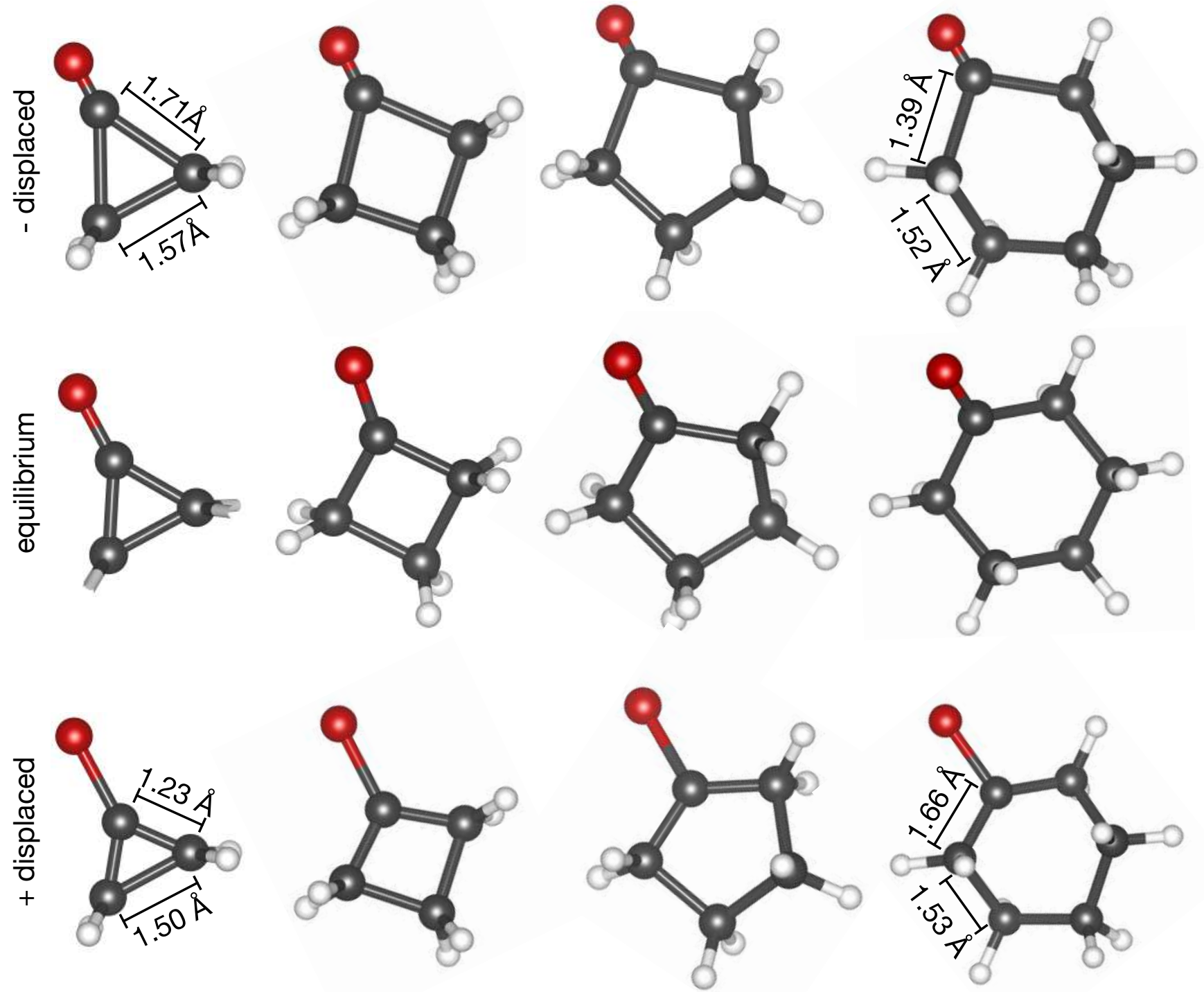

Figure 10: Snapshots taken from the animations provided in our web app. In smaller rings, the $\mathrm{C}=\mathrm{O}$ stretching frequency is higher due to a larger force constant. This can be thought of as being due the larger relative compression of $\mathrm{C}-\mathrm{C}$ bonds requires in the case of small rings. To highlight this effect, we give the bond lengths of a neighboring $\mathrm{CC}$ bond for the displacement of \pm the mode vector, i.e., the starting and end frames of the animation. Importantly, this figure also illustrates that the compression of the second nearest neighbor bonds is much more pronounced for the small rings than for the larger ones. 
<smiles>O=C(O)c1ccccc1O</smiles>

exp. $1662 \mathrm{~cm}^{-1}$ XTB $1780 \mathrm{~cm}^{-1}$<smiles>COC(=O)c1ccccc1C</smiles>

exp. $1737 \mathrm{~cm}-1$ XTB $1749 \mathrm{~cm}^{-1}$<smiles>NC(=O)c1ccccc1</smiles>

exp. $1661 \mathrm{~cm}^{-1}$ xTB $1737 \mathrm{~cm}^{-1}$<smiles>CN(C)C(=O)c1ccccc1</smiles>

exp. $1626 \mathrm{~cm}^{-1}$ XTB $1611 \mathrm{~cm}^{-1}$

Figure 11: Comparing the simulated carbonyl frequencies with the ones reported in the Spectral Database for Organic Compounds (SDBS). ${ }^{19}$ The top row of molecules, contain functional groups that form hydrogen bonds involving the carbonyl group in molecular packing - which impacts the stretching frequency. 14

system sizes one often has to deal with (e.g., in the case of metal-organic frameworks) no longer make it possible to get an on-the-fly result from a webservice but will rather require coupling with a dedicated simulation infrastructure (similar to what we recently proposed $^{20}$ ). As an alternative, one might provide on-the-fly estimates using a machinelearning model.

\section{Conclusions}

We presented an interactive web tool that allows simulating IR spectra for arbitrary molecules, which can then interactively be explored. This app can effectively be used to teach key concepts and intuitions on IR spectroscopy, but also to aid the interpretation of spectra in a research setting. We envision that the development of modular digital tools such as the one presented in this work has the potential to change how we do and teach chemistry. With the same tooling, we can aid researchers in analyzing the data and allow students to explore core concepts interactively. In light of the digital future of chemistry, investing in such tools seems like a forward-looking investment. 


\section{Methods}

For our web tool, we integrate a webservice ${ }^{21}$ for the simulation of IR spectra with an interactive frontend that can be used from a dedicated site as well as from an open-source electronic lab notebook (ELN). ${ }^{\mid 22}$ This design makes it possible to maintain and reuse the web service independent from the frontend - which in our case is also written in another programming language.

\section{Web service backend}

We use the FastAPI framework (https://fastapi.tiangolo.com/) to expose a representational state transfer (REST)-API for an IR simulation service that accepts SMILES as well as molfiles (see https://ir.cheminfo.org/v1/docs for documentation), using the cross-origin request (CORS) middleware to allow requests from other domains. Using the input molecule, we first perform a short conformer search, enhanced with the third version of the experimental torsion knowledge distance geometry method proposed by Riniker and Landrum ${ }^{23 \sqrt[24]{24}}$ as implemented in the RDKit. ${ }^{25}$ We then perform an energy minimization using the UFF force field ${ }^{26}$ and pick the lowest energy conformer for the next steps.

Then, we pass the molecule to an energy minimization followed by a calculation of the vibrational frequencies, for which we use the Atomistic Simulation Environment (ASE) ${ }^{27}$ as a high-level interface. For the energy minimization and the calculation of the vibrational frequencies, we give the user the choice between the generic GFNFF force-field, 28 that was fitted and benchmarked on a wide variety of chemistry, as well as the GFN-xTB extended tight-binding methods. ${ }^{29 / 30}$ We chose those techniques as they allow for accurate predictions for common organic molecules for low computational cost. More accurate, and expensive, simulations could be integrated — as we have recently shown ${ }^{[20}$ — via a dedicated simulation platform. To increase computational efficiency, outputs are disk- 
cached using the python-diskcache library. ${ }^{31}$ The code for the webservice, as well as a prebuilt Docker image, are available from https://github.com/cheminfo-py/xtbservice. The service is deployed from https://go.epfl.ch/learnir.

\section{Selecting atoms for highlighting}

To build a useful interactive frontend it is important to automatically select the atoms that are most "relevant" for a given mode. This then allows the frontend to highlight these atoms and provide mouse-over effects. However, always selecting the $k$ most displaced atoms is not a useful rule. Additionally, one would like to automatically select all atoms for translational modes. For the same reason, also a criterion on the relative contribution to the total absolute displacement of all atoms does not work well. For this reason, we heuristically select all atoms for which the Euclidean norm of their displacement $\|\mathbf{d}\|_{2}$ is greater than

$$
t=0.4 \max \left(\operatorname{diff}\left(\|\mathbf{d}\|_{2}\right)\right),
$$

where the diff operator returns the distance between neighboring elements. We use a similar criterion for determining the relevance of the normal modes for each bond. In this case, we replace the displacement with the Euclidean distance between the bond vectors. Note that for all displacement calculations we correct for the movements of the center of mass. Additionally, we also mask out the rotational/translational modes when we compute the information that is used for the highlight and mouse-over features. (We also investigated "keefinding" algorithms. ${ }^{32}$ But found our simple heuristic to work best.)

\section{Frontend}

The interactive frontend is implemented using the visualizer library. (https://github. com/NPellet/visualizer). ${ }^{33}$ This library allows to serialize the complete definition of the frontend, including all the interactions, in one json file. The vibrational modes are 
visualized with JSMol, the HTML5 modality of Jmol (http://jmol. sourceforge.net/),, 34 which provides numerous functions, including the options to perform distance measurements. The chemical structure editor is based on the JavaScript port ${ }^{[35}$ of the OpenChemLib library (https://github.com/actelion/openchemlib). ${ }^{36}$ The frontend is on https: //go.epfl.ch/learnir. Upon first use, users will be guided through the app using a onboarding tour we created using the shepherd library (https://shepherdjs.dev/).

\section{Creating new structure sets}

The frontend we developed allows creating a custom set of molecules for which the IR spectra can be compared. By default, we provide some sets to illustrate some key electronic effects but teachers can easily create their own sets in the "Series" layer of the web app. There, an arbitrary number of collections of arbitrary molecules can be created which will be stored in the json file defining the frontend using the OpenChemLib ${ }^{35}$ ID.

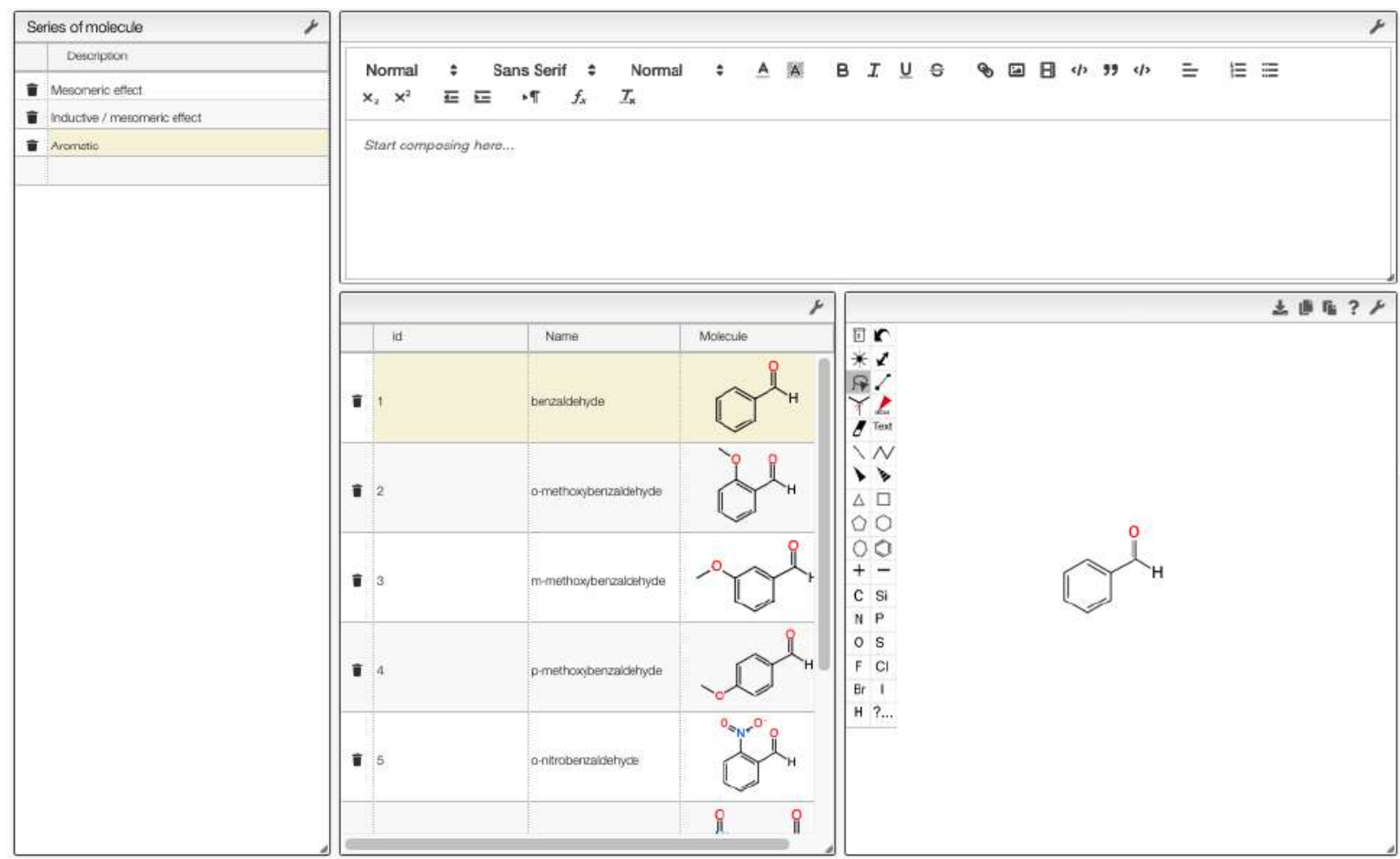

Figure 12: Teachers can create custom sets of molecules. Since the requests are cached by the simulation service, the responses for these sets will be particularly fast. 
A more extensive discussion of the technical background is provided on the documentation page of the app (https://docs.c6h6. org/docs/eln/uuid/10b6a7229db7dd815afcc75e77c2d6cd). Additionally, we provide a docker-compose recipe that allows deploying the full system on any environment (github .com/cheminfo-py/ir-teaching-app) via a Ngnix web server. ${ }^{37}$ In practice, this means teachers only need to follow our instructions and can host a customized version of the app on their own computer/server. Given that only two command are needed (git clone, docker-compose up --build -d) one could even encourage motivated students to run it on their own computer.

\section{Acknowledgement}

We thank the cheminfo developer community for their contributions. The research from K.M.J. and B.S. was supported by the Swiss National Science Foundation (SNSF) under Grant 200021_172759.

\section{References}

(1) Woodward, R. B. BOOK REVIEWS. Journal of the American Chemical Society 1950, 72, 3327.

(2) Frank, M.; Bäumer, M.; Kühnemuth, R.; Freund, H.-J. Metal Atoms and Particles on Oxide Supports: Probing Structure and Charge by Infrared Spectroscopy. The Journal of Physical Chemistry B 2001, 105, 8569-8576.

(3) Bennett, J.; Forster, T. IR Cards: Inquiry-Based Introduction to Infrared Spectroscopy. Journal of Chemical Education 2009, 87, 73-77. 
(4) Heuer, W. B.; Koubek, E. An Investigation into the Absorption of Infrared Light by Small Molecules: A General Chemistry Experiment. Journal of Chemical Education 1997, 74,313 .

(5) Hill, M. A. Infrared Spectroscopy in the General Chemistry Lab. Journal of Chemical Education 2001, 78, 26.

(6) Borgsmiller, K. L.; O'Connell, D. J.; Klauenberg, K. M.; Wilson, P. M.; Stromberg, C. J. Infrared and Raman Spectroscopy: A Discovery-Based Activity for the General Chemistry Curriculum. Journal of Chemical Education 2012, 89, 365-369.

(7) Adams, W.; Sonntag, M. D. Vibrational Spectroscopy of Hexynes: A Combined Experimental and Computational Laboratory Experiment. Journal of Chemical Education 2018, 95, 1205-1210.

(8) Thrall, E. S.; Lee, S. E.; Schrier, J.; Zhao, Y. Machine Learning for Functional Group Identification in Vibrational Spectroscopy: A Pedagogical Lab for Undergraduate Chemistry Students. Journal of Chemical Education 2021,

(9) Dunnagan, C. L.; Dannenberg, D. A.; Cuales, M. P.; Earnest, A. D.; Gurnsey, R. M.; Gallardo-Williams, M. T. Production and Evaluation of a Realistic Immersive Virtual Reality Organic Chemistry Laboratory Experience: Infrared Spectroscopy. Journal of Chemical Education 2019, 97, 258-262.

(10) Hollas, J. Modern spectroscopy; Wiley: Chichester, 2004.

(11) Atkins, P. W. Atkins' Physical chemistry; Oxford University Press: Oxford, United Kingdom New York, NY, 2018.

(12) McQuarrie, D. Physical chemistry : a molecular approach; University Science Books: Sausalito, Calif, 1997.

(13) Sweller, J.; Ayres, P.; Kalyuga, S. Cognitive Load Theory; Springer New York, 2011. 
(14) McEvoy, J. P. Characterizing Carbonyls with Infrared Spectroscopy: An Introductory Chemistry Experiment in a Molecular Bioscience Program. Journal of Chemical Education 2014, 91, 726-729.

(15) Hammett, L. P. The Effect of Structure upon the Reactions of Organic Compounds. Benzene Derivatives. Journal of the American Chemical Society 1937, 59, 96-103.

(16) Rzepa, H. Why Is the Carbonyl IR Stretch in an Ester Higher than in a Ketone? Henry Rzepa's Blog. https:/ /www.ch.imperial.ac.uk/rzepa/blog/?p=9606, 2013.

(17) Baeyer, A. Ueber Polyacetylenverbindungen. Berichte der deutschen chemischen Gesellschaft 1885, 18, 2269-2281.

(18) Keeler, J. Chemical structure and reactivity : an integrated approach; Oxford University Press: New York, NY, 2014.

(19) of Advanced Science, N. I.; Technology, SDBS Web. 2021,

(20) Jablonka, K. M.; Zasso, M.; Patiny, L.; Marzari, N.; Pizzi, G.; Smit, B.; Yakutovich, A. V. Connecting lab experiments with computer experiments: Making "routine" simulations routine. ChemRxiv, 10.33774/chemrxiv-2021-h3381-v2 2021,

(21) Patiny, L.; Borel, A. ChemCalc: A Building Block for Tomorrow's Chemical Infrastructure. Journal of Chemical Information and Modeling 2013, 53, 1223-1228.

(22) Patiny, L.; Zasso, M.; Kostro, D.; Bernal, A.; Castillo, A. M.; Bolaños, A.; Asencio, M. A.; Pellet, N.; Todd, M.; Schloerer, N.; Kuhn, S.; Holmes, E.; Javor, S.; Wist, J. The C6H6 NMR repository: An integral solution to control the flow of your data from the magnet to the public. Magnetic Resonance in Chemistry 2017, 56, 520-528.

(23) Riniker, S.; Landrum, G. A. Better Informed Distance Geometry: Using What We Know To Improve Conformation Generation. Journal of Chemical Information and Modeling 2015, 55, 2562-2574. 
(24) Wang, S.; Witek, J.; Landrum, G. A.; Riniker, S. Improving Conformer Generation for Small Rings and Macrocycles Based on Distance Geometry and Experimental Torsional-Angle Preferences. Journal of Chemical Information and Modeling 2020, 60, 2044-2058.

(25) Landrum, G. et al. rdkit/rdkit: 2021_03_5 (Q1 2021) Release. 2021; https://zenodo. org/record/5242603.

(26) Rappe, A. K.; Casewit, C. J.; Colwell, K. S.; Goddard, W. A.; Skiff, W. M. UFF, a full periodic table force field for molecular mechanics and molecular dynamics simulations. Journal of the American Chemical Society 1992, 114, 10024-10035.

(27) Larsen, A. H. et al. The atomic simulation environment-a Python library for working with atoms. Journal of Physics: Condensed Matter 2017, 29, 273002.

(28) Spicher, S.; Grimme, S. Robust Atomistic Modeling of Materials, Organometallic, and Biochemical Systems. Angewandte Chemie International Edition 2020, 59, 15665-15673.

(29) Bannwarth, C.; Ehlert, S.; Grimme, S. GFN2-xTB-An Accurate and Broadly Parametrized Self-Consistent Tight-Binding Quantum Chemical Method with Multipole Electrostatics and Density-Dependent Dispersion Contributions. Journal of Chemical Theory and Computation 2019, 15, 1652-1671.

(30) Grimme, S.; Bannwarth, C.; Shushkov, P. A Robust and Accurate Tight-Binding Quantum Chemical Method for Structures, Vibrational Frequencies, and Noncovalent Interactions of Large Molecular Systems Parametrized for All spd-Block Elements ( $\mathrm{Z}$ =1-86). Journal of Chemical Theory and Computation 2017, 13, 1989-2009.

(31) Jenks, G. DiskCache: Disk Backed Cache. 2021; https://github.com/grantjenks/ python-diskcache/tree/master. 
(32) Satopaa, V.; Albrecht, J.; Irwin, D.; Raghavan, B. Finding a "Kneedle" in a Haystack: Detecting Knee Points in System Behavior. 2011 31st International Conference on Distributed Computing Systems Workshops. 2011.

(33) Pellet, N. jsGraph and jsNMR-Advanced Scientific Charting. Challenges 2014, 5, 294-295.

(34) Hanson, R. M. Jmol- a paradigm shift in crystallographic visualization. Journal of Applied Crystallography 2010, 43, 1250-1260.

(35) Zasso, M.; Cheminfo-Bot,; Patiny, L.; Rufenerc,; Greenkeeper,; Castillo, A.; Kostro, D.; Fidler, R. cheminfo/openchemlib-js: openchemlib v7.4.2. 2021; https://zenodo.org/ record/5165170,

(36) Sander, T.; Freyss, J.; von Korff, M.; Rufener, C. DataWarrior: An Open-Source Program For Chemistry Aware Data Visualization And Analysis. Journal of Chemical Information and Modeling 2015, 55, 460-473.

(37) Reese, W. Nginx: The High-Performance Web Server and Reverse Proxy. Linux J. 2008, 2008. 
Graphical TOC Entry

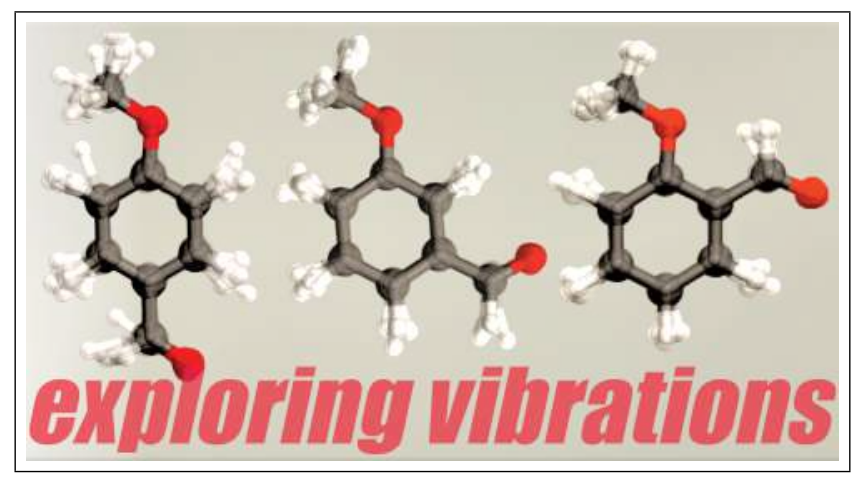

